\title{
Single-cell transcriptome reveals a testis-specific expression profile of TCEA in human spermatogenesis
}

\author{
Pankaew, S. ${ }^{1 \dagger}$ and Pramoj Na Ayutthaya, P. ${ }^{2 \dagger *}$
}

'Aix-Marseille Université, CNRS, INSERM, CIML, I 3009 Marseille, France

${ }^{2}$ Department of Molecular Biology, Max Planck Institute for Developmental Biology, 72076 Tübingen,

Germany

$\dagger=$ equal contribution

$*=$ corresponding author

Contact information

Pankaew, S. = pankaewf@gmail.com

Pramoj Na Ayutthaya, P. = pr.pramoj@gmail.com

\section{Abstract}

Transcription elongation factor A (TCEA) is a eukaryotic transcriptional molecule, required for a formation of initiation and elongation of gene transcription-mediated RNA polymerase II (RNAPII) complex, to promote transcription-coupled nucleotide excision repair (TC-NER) after RNAPII backtracking recovery. TCEA shares three isoforms in which TCEAI is ubiquitously expressed among all eukaryotic cells. We found a spermatogenesis TCEAI and TCEA2 expression profile has a unique transcriptional programme, compared with embryogenesis. Moreover, the testis-specific TCEA2 profile correlates with gene transcription, whereas TCEAI specifically correlates with genes transcribed for Nuclear excision repair (NER) during human spermatogenesis. We also found that the expression activation of RNF20, a TCEA/ inhibitor, leads to expressional TCEA/ reduction, but having no direct impact on TCEA2 expression, implying the potential RNF20-dependent transcriptional switching of TCEA2 in transcriptional regulation during spermatogenesis. Our analysis defined a transcriptional bursting event where transcription-coupled repair (both Base excision repair and Nuclear excision repair) is a major pathway highly expressed in early spermatogenesis, supporting the transcriptional scanning hypothesis of which mutation of transcribed genes is effectively repaired as proposed by Xia B., et al. (2020). 


\section{Introduction}

Transcriptional bursting is a fundamental biological process signatured in developments such as murine embryogenesis and spermatogenesis (Li et al., 20I3; Ochiai et al., 2020). Upon the transcription, massive transcription factors are expressed to help promote downstream transcribed genes (Rhee et al., 2008). Transcription factor IIS (TFIIS) or transcription elongation factor $A$ (TCEA) is one of the most crucial molecule to coordinate gene transcription via forming an initiation and elongation complex with RNA polymerase II (RNAPII) (Guglielmi et al., 2007; Kim et al., 2007; Prather et al., 2005; Reines et al., 1989) and also to help safeguard transcriptional error-prones from RNAPII complex by possessing a helicase activity that dissociates mismatched transcribed DNA lesion via mRNA cleavage of transcription-coupled nuclear excision repair or TC-NER (Kalogeraki et al., 2005; Kettenberger et al., 2003; Sigurdsson et al., 2010). Among Chordata phylum, TCEA shares three isoforms: TCEAI, TCEA2, and TCEA3 and only TCEA/ has been investigated at biochemical level. Previous study demonstrated that the RNAPII complex with TCEAI can help recover RNAPII backtracking (Lisica et al., 2016) upon promoting a bypass via a UV-induced 8-oxoguanine presence (Kuraoka et al., 2007). Yet it is unclear whether other isoforms share a similar expression profile as TCEAI and how those isoforms biochemically regulate downstream pathways. Eukaryotic TCEA is also crucial for biological developments across kingdoms ranging from yeast, plant to animal (Cha et al., 20I3; Grasser et al., 2009; Guglielmi et al., 2007; Park et al., 20I3; Yang et al., 2018). Dysfunction of TCEA/ significantly leads to cancer proliferation, embryonic lethality, and sperm abnormality in human (Horiuchi et al., 20I8; Ito et al., 2006; Shema et al., 20I I) due to severely reduced gene transcriptions and genome stability (Lennon et al., 1998; Zatreanu et al., 2019). A recent work from Xia B., et al. (2020) has demonstrated that SNP variants in transcribed genes have significantly lowered mutation rates rather than those in non-transcribed genes, suggesting the gene repairs can be fine-tuned via transcription-coupled repair during gene transcription in spermatogenesis (Xia et al., 2020).

Intriguingly, expression patterns of TCEA/, TCEA2, and TCEA3 showed a unique characteristic in Xenopus embryogenesis (Labhart and Morgan, 1998), hypothesizing that these three isoforms may have functional preferences for both gene transcription and DNA repair based upon their gradient expressions among each cell stage. TCEA2 was reported, in 1997 to be testis-specific gene (Weaver and Kane, 1997). But how TCEA2 is quantitatively expressed at single-cell level is not known. In this work, we sought to investigate if TCEA/, TCEA2, and TCEA3 expressions can be correlated with gene transcription and DNA repair expression in 
human spermatogenesis. Collectively, our study reports the germline-specific TCEAI and TCEA2 transcription in which the expressed isoforms can refer to the functional preference of gene transcription and the repair. This analysis would help highlight a new outlook of expression-based TCEA isoforms, which infer a new mechanism of RNF20-independent TCEA2 regulation in the context of developmental biology.

\section{Result and Discussion}

\section{Transcription elongation factor TCEA/ and TCEA2 expression profiles correlate with cellular gene transcription during spermatogenesis}

A previous study from Xia B., et al. (2020) generated a hypothesis "transcriptional scanning" in which expressed genes can be effectively repaired via a well-known characteristic of transcription-coupled repair (TCR) and that can promote an impact of gene mutations of non-transcribed genes in mammalian testes (Xia et al., 2020). We here investigated what molecular factors contributing to the transcription and the repair can be a characteristic of fixing spermatogenesis-expressed gene mutations at gene transcription level. To test dataset robustness and reproducibility, we performed an independent statistical analysis and identified the specific cell types (Figure IA) by using gene markers from human testes atlas (Supplementary Table I). Overall, our statistical analysis provided similar results given by principal components embedding and identified groups of spermatogenesis-related genes which are highly expressed among individual cell stages by comparing with known marker genes (Figure IB\&Supplementary Figure IA\&IB) as similar to the human testes atlas study (Guo et al., 2018). Lists of germline-selective differentially expressed gene clusters are provided in Supplementary Table I. Human gene transcription is highly expressed in round spermatid (RS) stage with low leakage of mitochondrial gene expression, suggesting the stage was not under stress-induced, but rather cell proliferation- or morphogenesis-induced scenario (Figure IB\&Supplementary Figure IC\&ID). We found that the expression profile of TCEAI and TCEA2 showed a specific pattern (Figure IC), in which during the early stage of spermatogenesis from $\mathrm{Sg}-\mathrm{I}$ to RS-I, TCEAl expression decreased as TCEA2 expression increased. Later, the expression TCEA/ were recovered during the stage RS-2 to ES-4. As expected, TCEA enhances transcription elongation to help increase gene transcription rate in complex with RNAPII (Schweikhard et al., 20I4a; Schweikhard et al., 20I4b). Interestingly, the 
bioRxiv preprint doi: $h t t p s: / / d o i . o r g / 10.1101 / 2021.03 .10 .434830$; this version posted March 11, 2021. The copyright holder for this preprint (which was not certified by peer review) is the author/funder, who has granted bioRxiv a license to display the preprint in perpetuity. It is made available under aCC-BY-NC 4.0 International license.

gradient pattern of TCEAI and TCEA2 shows a unique characteristic only observed in spermatogenesis not as in developmental and tissue-specific characteristic in embryogenesis from humans (Supplementary Figure 2) and from Xenopus (Labhart and Morgan, 1998; Sladitschek et al., 2020).

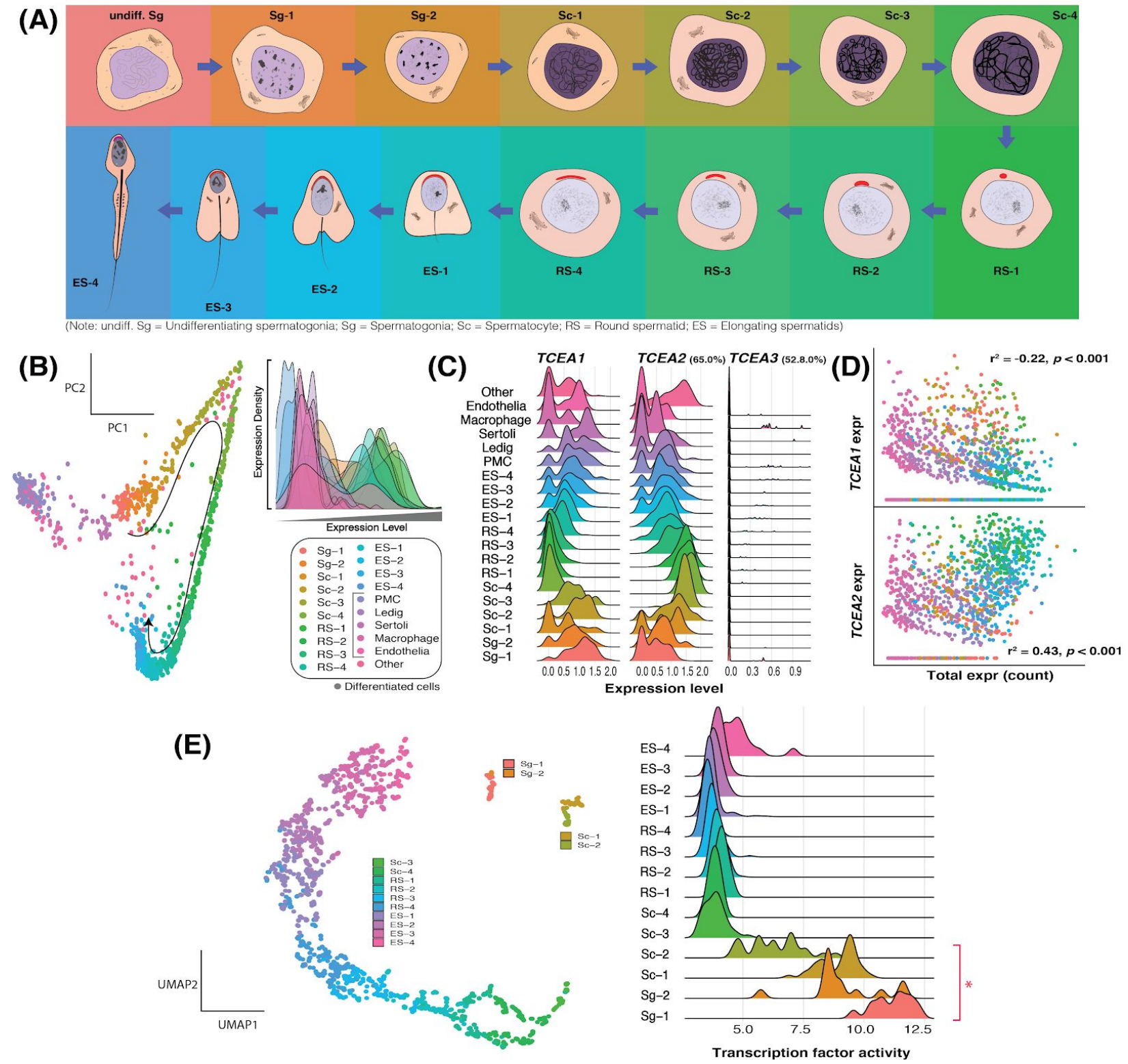

Figure I. Gradient expression of TCEA/ and TCEA2 with cellular gene transcription upon developmental niches of spermatogenesis. (A) Schematic spermatogenesis-related germlines given in the analysis. (B) Principal component analysis (PCA) of scRNA dataset provided by Xia B., et al. (2020) shows cell type-specific trajectories upon gene transcription in spermatogenesis with total gene transcription profiles of each cell type in humans. (C) Transcriptional profiling of human TCEAI, TCEA2 and TCEA3 given protein sequence identity $65.0 \%$ of TCEA2 and $52.8 \%$ of TCEA3, compared with TCEAI in individual cell types. (D) TCEA/ and TCEA2 expression count correlates statistically with gene transcription in spermatogonial-specific germlines. (E) 
Profiling of transcription factor activity in human spermatogenesis (high activity from Sg-I to Sc-2 as indicated with red asterisk) provides unique characteristics of transcription factors involved in the spermatogenesis given by uniform manifold approximation and projection (UMAP, left) and quantitative activity profile (right). [all statistical analysis provided in the figure was tested by Pearson's correlation at a cut-off $p$-value $=0.00 \mathrm{I}]$

TCEA is also contributed to help balance ribosomal gene transcription under cell stress (Gomez-Herreros and de Miguel-Jimenez, 20I2), but that is not likely our observation because mitochondrial gene leakage as inferred to cell stress is low during spermatogenesis (Supplementary Figure ID). Next, we found that gradient total gene transcription of each stage ranging from spermatogonia $(\mathrm{Sg})$ to round spermatid (from low to high) correlates negatively with TCEA/ and positively with TCEA2, but not as similar case in human embryogenesis (Figure ID\&Supplementary Figure 2). Therefore, the expression profile of TCEA/ and TCEA2 can be unique in human spermatogenesis. Based on assumptions: (i) total encoding gene being transcribed is mediated by RNAPII in complex with TCEA, and (ii) each isoform of TCEA can be translated into functional TCEA. We reasoned that the molecular testis-specific TCEA2 preference after translation can promote transcription regulations due to "transcriptional TCEA switching" over other isoforms for spermatogenesis-related gene transcription homeostasis. To investigate transcriptional activity profiling during the spermatogenesis, we embedded the uniform manifold approximation and projection of the transcription factor expressions of which the relevant genes shared their TF motifs are given high expression scores to infer the TF or regulon activity. We observed high regulon activities and unique trajectories during the early spermatogenesis (from Sg-I to Sc-2), compared with the rest of cell stages that share the continuous trajectories (Figure IE). That suggests that the early spermatogenesis has a unique transcriptional mechanism and regulation to maintain important gene expressions prior to initialising round and elongating spermatid differentiation.

\section{Nuclear excision repair is a major DNA repair expressed during human} spermatogenesis

We calculated regulon activities using Single-Cell rEgulatory Network Inference and Clustering (SCENIC) (Van de Sande et al., 2020), a computational method for inferring gene regulatory network and its activities. The inferences calculated co-expression between a transcription factor and its targets, clustered them into a 'regulon', and computed the regulon activities from the target genes in single-cell. We found high regulon activities in the early spermatogenesis (from Sg-I to Sc-3) with high number of regulon interactions, compared to 
other cell stages (Figure IE\&Figure 2A). To investigate downstream gene functions regulated by the relevant regulons, we performed gene ontology (GO) analyses with an adjusted $p$-value cutoff at $0.0 \mathrm{I}$. List of the regulons and downstream genes identified in our analyses is provided in Supplementary Table 2\&Supplementary file I. Both positive and negative regulations of RNAPII-mediated transcription were shown prominent during the Spermatogonia and Spermatocyte (only Sc-I and Sc-2), while Sc-3 has an unclassified regulation of the transcription (Figure 2B). However, positive regulation of the transcription regulation was not found in other cell stages (Supplementary Figure 3). This suggests that high positive activity of the RNAPII-mediated gene transcription, so called "transcriptional bursting" occurs in the specific early stages of the spermatogenesis from Sg-I to Sc-3. Given the transcriptional scanning hypothesis, transcriptional bursting may promote gene repairs to fine-tune gene adaptations due to high transcription rate and transcription-coupled repair expression (Svejstrup, 2002; Xia et al., 2020). We therefore performed correlation analyses of the regulon activity and each of the DNA repair pathways. We found that both Base excision repair (BER) and Nuclear excision repair (NER) show high correlations with the regulon activity, suggesting that preferential transcription-coupled repair mediates transcribed gene repairs during the transcriptional bursting (Figure 2C\&Supplementary Figure 6A). In post-transcriptional bursting, the NER pathway showed the highest expression among other DNA repair pathways (Figure 2D\&Supplementary Figure 6B). 


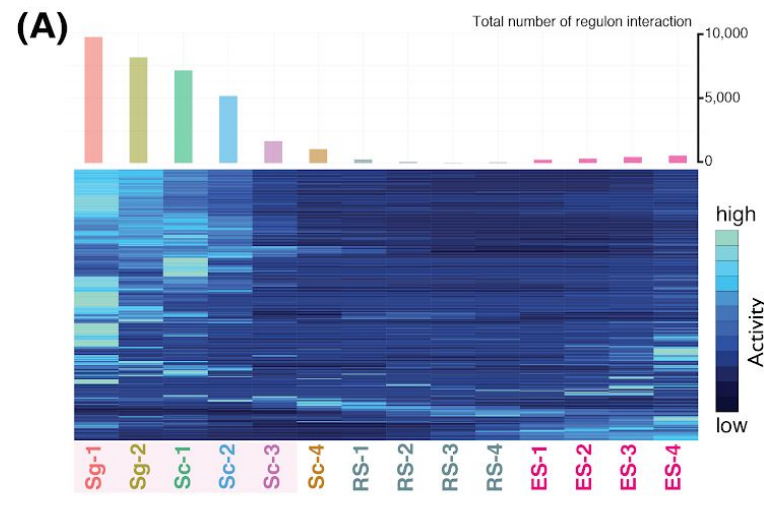

(B)
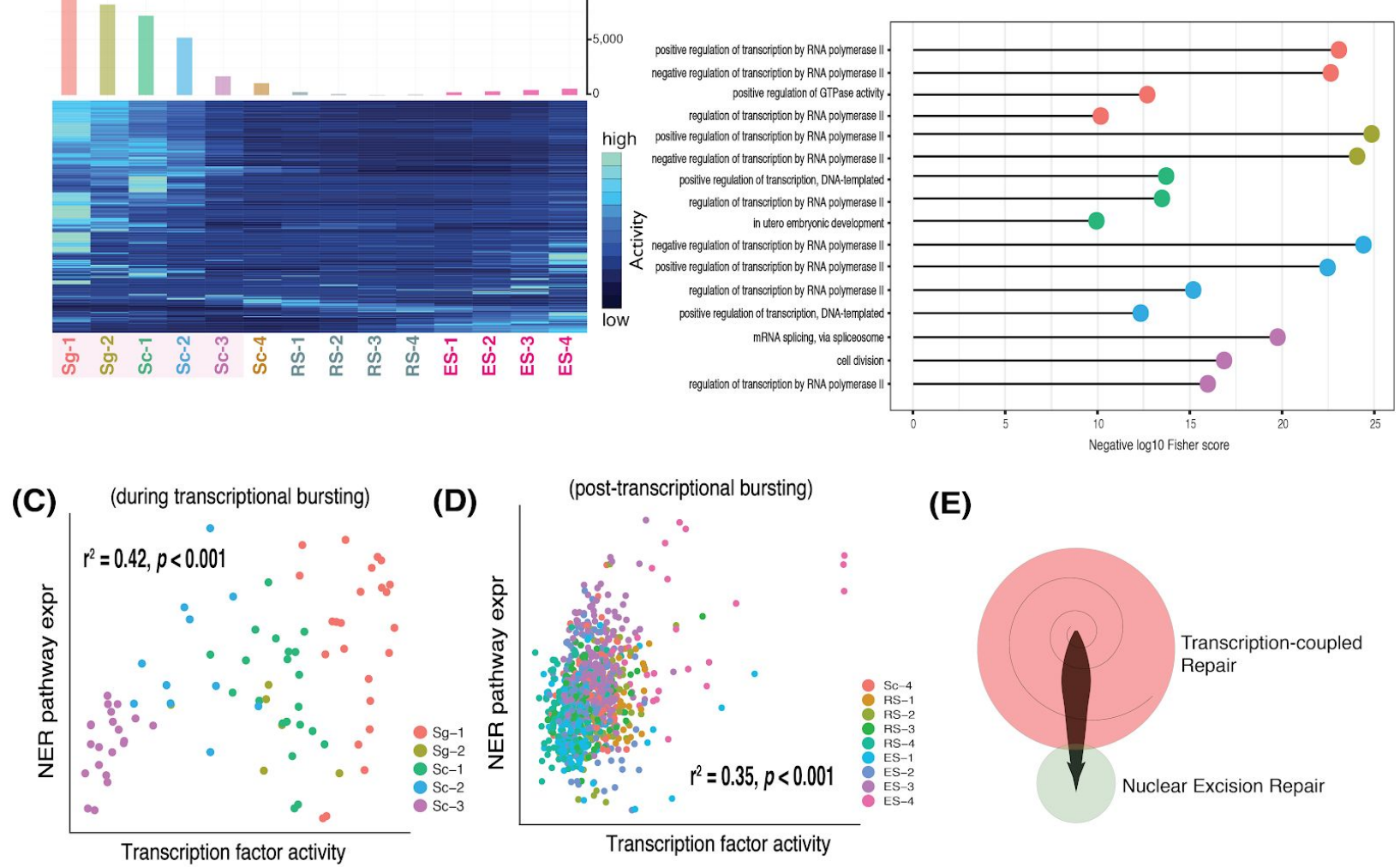

(E)

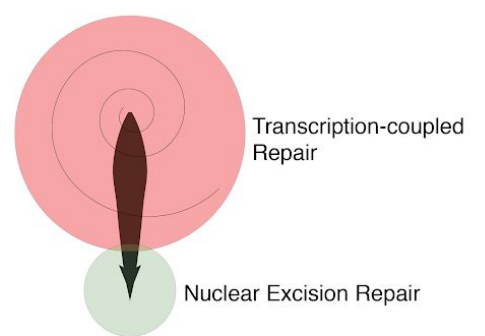

Figure 2. Defining transcriptional bursting during human spermatogenesis, which shares a high correlation with nuclear excision repair (NER) expression. (A) Heatmap representation of regulon activity profiling of each cell stage during spermatogenesis with a number of regulon-targeted gene networks represented in the histogram. (B) Selected gene ontology (GO) analysis of genes highly regulated by regulons involved in RNAPII-mediated gene transcription in Sg-I, Sg-2, Sc-I, Sc-2, and Sc-3 represented by colours given from the heatmap. (C) Correlation analysis of NER pathway expression with transcriptional bursting activity by the PySCENIC. (D) Correlation analysis of NER pathway expression with transcription activity after the bursting by the pySCENIC. (E) Schematic model represents NER as a major DNA repair facilitating transcribed gene repair during the transcriptional scanning (both in the transcriptional bursting; red, and the post- transcriptional bursting; light green) as proposed by Xia B., et al. (2020). [correlation analyses were tested by Pearson's correlation with a cut-off $p$-value $=0.001$; gene ontology $(\mathrm{GO})$ analyses were tested by Fisher's exact test with a cut-off $p$-value $=$ $0.01]$

The analysis here indicated an important biological regulation upon the transcriptional scanning scenario. Transcriptional-coupled repair is co-expressed with the coupling transcription activity during the early cell stage ( $\mathrm{Sg}-\mathrm{I}$ to $\mathrm{Sc}-3$ ) of the human spermatogenesis, whereas NER is primarily co-expressed during the post-transcriptional bursting as shown in the hypothetical model (Figure 2E). This transcriptional bursting event is likely cell-stage specific regulation during the human germline development and highly involved with the transcriptional scanning event because the transcription-coupled repair is responsible for reducing gene mutations during which high activities of RNAPII-mediated gene transcription. Overall, the 
transcriptional bursting in the early spermatogenesis is potentially responsible for the transcriptional scanning hypothesis in which the transcription-coupled repair plays a major role in reducing mutations of transcribed genes and promoting gene adaptations.

\section{TCEA / expression infers preferential transcription involved in transcription-coupled repair in human spermatogenesis}

We next investigated TCEAI and TCEA2 expression, which may transcriptionally correlate with the spermatogenesis-related NER expression. We realized that having a single marker to determine each of the DNA repair pathways would introduce less plausible pathway expressions due to high variants of single-cell expression counts. Therefore, we curated gene lists grouped into individual gene clusters, representing unique DNA repair characteristics via our knowledge-based approach. List of genes involved in the DNA repair pathways is provided in Supplementary Table 3. Albeit nuclear excision repair (NER) is biochemically classified into two pathways: TC-NER and general genome NER (GG-NER), it is difficult to interpret whether TC-NER is more highly expressed than GG-NER given in our dataset without protein activity. We then created the NER-related gene cluster to infer TC-NER/GG-NER expression, which is highly expressed in Sc stage, with the presence of specific TC-NER markers such as POLRA2, $E R C C 6$ or ERCC8 and XPC, respectively (Supplementary Figure 4A,4B,4C,4D and Supplementary Figure 5). We found that only TCEA/ has the highest correlation with NER pathway, but not in the case of TCEA2. We also plotted TCEA/ and TCEA2 against other DNA repair expression profiles (Figure $3 A$ ). Although all DNA repair pathways were highly expressed during the early spermatogenesis, neither TCEA/ nor TCEA2 showed strong correlations with Base excision repair (BER) and Mismatch repair (MMR) (Supplementary Figure 4E\&4F\&Supplementary Figure 5), implying only TCEA/-mediated TC-NER during gene transcription.

Messenger RNA from gene transcription can be implemented to facilitate gene repairs via NHEJ mechanism during gene transcription (Chakraborty et al., 2016). That would also speculate that mRNA products can be utilized to repair transcribed genes via NHEJ as we observed a positive correlation between TCEA2 and NHEJ pathway with high cellular gene expressions (Supplementary Figure IC\&4F). We also found that NER pathway is highly expressed given high expressions among POLR2A, ERCC6, and ERCC8, but low XPC expression, implying the correlation of TCEA/ expression infers the TC-NER pathway activity (Figure 3A\&Supplementary Figure 4). Moreover, a previous report demonstrated that ERCC6, 
which encodes Cockyne Syndrome B (CSB), crucially insures a preferential DNA repair during the G2/M-phase of cell division, leveraging transcription-coupled related homologous recombination or proposed TC-HR in transcriptionally active chromatin (Aymard et al., 20I4; Batenburg et al., 20I5). In order for "pushing" a stalled CSB-RNAPII complex, TCEA is required to recover the RNAPII-mediated transcription elongation. Speculatively therefore, TC-HR would need a required amount of TCEA to facilitate the repair as we observed the positive correlation between TCEAI and HR pathway during spermatogenesis (Supplementary Figure 4E). TCEA/ expressed during Homologous recombination (HR) while TCEA2 expressed during Non-homologous end-joining (NHEJ) and Inter-crosslinking repair (ICL), which also highly expressed and involved in S-phase (Supplementary Figure 7) in which DNA replication and ICL repair is a crucial prerequisite to fix mutagenic lesions at a replication fork for the cell cycle progression in the early and mid stage (Sg to RS) of spermatogenesis (Supplementary Figure 4\&5). Given a high scoring expression of spermatogenesis G2/M-phase during cell cycle (Supplementary Figure 7), we found that cell division-relevant DNA repairs such as HR and NHEJ were highly cooperated during the early spermatogenesis, but more often expressed for only NHEJ during RS stage (Supplementary Figure 4\&6) because NHEJ is more efficient and faster to facilitate DSB repair, but less accurate than HR for genomic stability (Bee et al., 20I3; Derbyshire et al., 1994; Mao et al., 2008; Shrivastav et al., 2008). We detected an increased expression of RNF20, a known inhibitor of TCEAl from the stages Sg-3 to RS-3 (Supplementary Figure IE). We also plotted TCEAI and TCEA2 against RNF20, a crucial inhibitor suppressing TFIIS activity in human cells for transcriptional TCEA balance. We found that TCEA/ has an antagonistic correlation, whereas TCEA2 has a synergistic correlation with $R N F 20$ profile during spermatogenesis (Figure $3 \mathrm{~B}$ ). Interestingly, RNF20 positively correlates with spermatogenesis-related gene transcriptions. This implies that $R N F 20$ selectively suppresses TCEA/ activity, but has no direct inhibition towards TCEA2 gene transcriptions during spermiogenesis (Sc-3 to ES-2). Thus, we proposed a hypothetical model in Figure 3C. Nonetheless, potential molecular suppression related to TCEA2 has to be further investigated. 

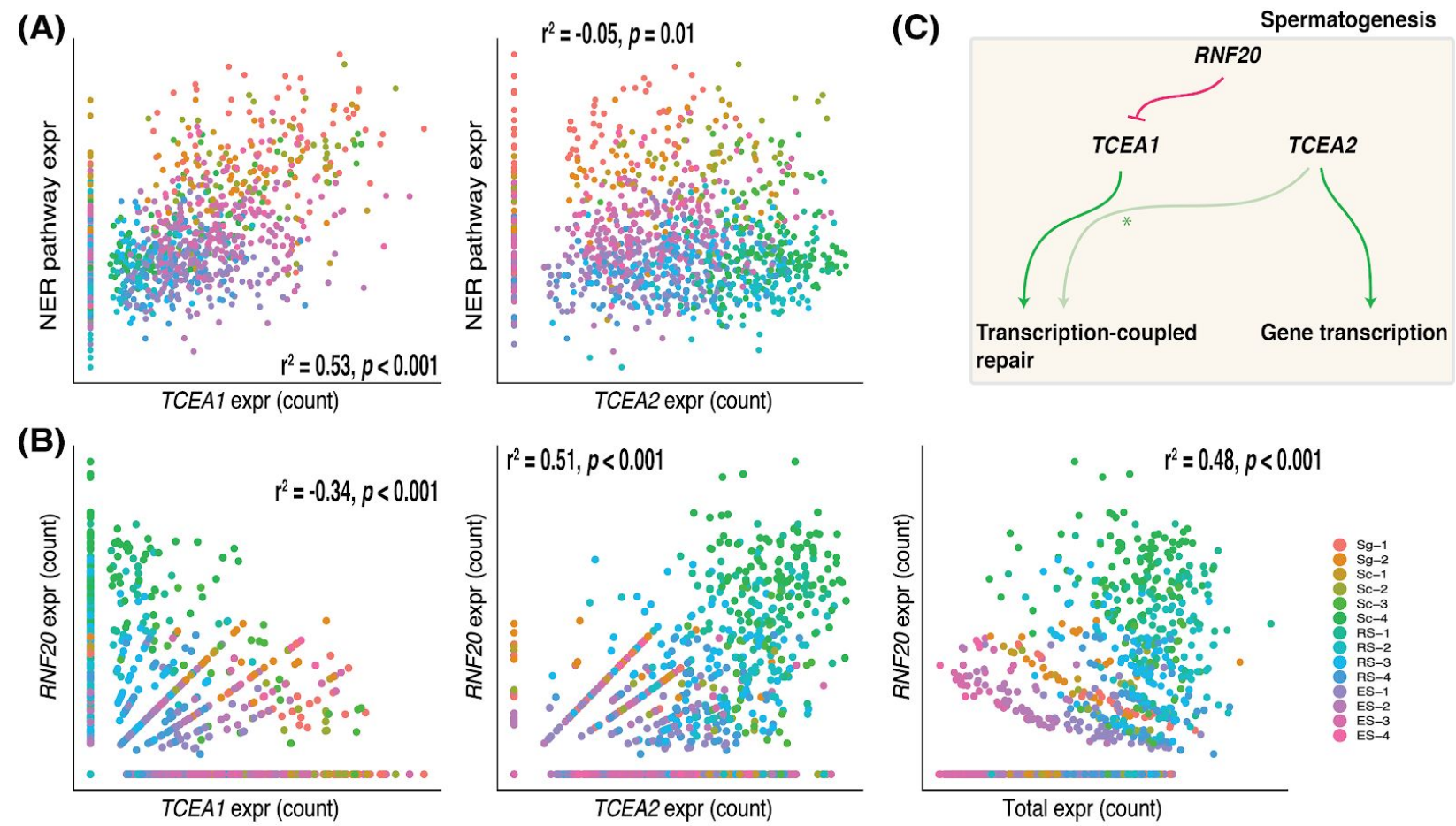

Figure 3. Comparative expression correlations between TCEA/ or TCEA2 and DNA repair pathways and analysis of DNA repair pathway expression in human spermatogenesis. $(A)$ Correlation analyses of NER pathway expression with TCEA / and TCEA2. (B) Correlation analyses of RNF20 regulations upon TCEAI (left), TCEA2 (middle) expression and gene transcription (right). (C) Proposed gene interaction of RNF20-mediated TCEAI and preferential TCEA switching based on transcription profiling [preference, green; inhibition, red; no correlation, asterisked light green]. [all statistical analysis provided in the figure was tested by Pearson's correlation at a cut-off $p$-value $=0.00 \mathrm{I}]$ 


\section{Conclusion}

Transcription elongation factor A, consisting encoded three isoforms of which the first isoform TCEAI plays crucial roles in transcription initiation and elongation, helps facilitate RNAPII-mediated gene transcription and repair. Despite well-studied TCEA/ functions, its isoform such as TCEA2 had been poorly investigated since 1997. Here, we investigated spermatogenesis-related expressional characteristics of individual TCEA isoform to help predict what potential molecular preferences would be whether involved in gene transcriptions and repairs by using a single-cell RNAseq data from Xia et al. (2020). We further employed a gene regulatory network inference method (PYSCENIC) to infer regulon activities during which the high presence of regulon activity so called "transcriptional bursting" occurred in the early spermatogenesis (Sg-I to Sc-3). We discovered that transcription-coupled repair expression (both NER and BER) is preferentially co-occurred during the transcriptional bursting, while NER is solely expressed in the post-bursting among other repair pathways. TCEAI is shown coexpressing with the NER pathway expression, known for having a helicase activity to facilitate RNAPII backtracking recovery. On the other hand, TCEA2, despite sharing a high protein sequence identity, has not been described to share NER regulation.

Interestingly, we showed that RNF20, the TCEAl inhibitor increases during the post-transcriptional bursting, resulting in the decrease of TCEA/ expression, while TCEA2 expression increases along with total gene transcription. With the specific expression profile of testis-specific TCEA and its correlation analyses, we propose a hypothetical model of the expressional isoform switching of which TCEA/ can be down-regulated by RNF20 after the transcriptional bursting stages, while allow TCEA2 to facilitate gene transcriptions during human spermatogenesis. Finally, our analysis also supports the transcriptional scanning hypothesis that transcription-coupled repair is highly expressed to help reduce gene mutations during the transcriptional bursting. 


\section{Methods}

\section{Single-cell dataset and analyses using Seurat}

Single-cell RNA-Seq and metadata were obtained from Xia B., et al. (2020). The data has already been integrated using Seurat (v3) integration process (Stuart et al., 2019). We performed data quality control by removing cells with less than 200 features expressed and features with less than 3 cells expressing total mRNA transcripts. We also filtered out cells with a percentage of mitochondrial genes $>10 \%$, which represents cells undergoing apoptosis (Osorio and Cai, 2020).

\section{Dimension reduction plot from principal components}

The integrated UMI counts were logarithmically normalized by using NormalizeData function in Seurat and scaled by multiplying by the scale factor of 10,000. From the normalized dataset, 2,000 highly variable expressing genes were selected by using a 'variance stabilizing transformation' (vst) from Seurat FindVariableFeatures. These features were then used to perform a principal components analysis (PCA) with all parameters in default. The first two principal components were then used to plot the embedded cells in 2-dimensional space.

\section{Pathway expression scoring from knowledge-based gene curation}

Gene sets involved in DNA repair pathways were manually curated from numbers of literature. We categorized these lists into 6 different pathways containing: Nuclear excision repair (NER), Base excision repair (BER), Mismatch repair (MMR), Homologous recombination (HR), Non-homologous end-joining (NHEJ), and Inter-crosslinking repair (ICL). The list of genes are provided in the Supplementary Table 2. The list of genes in each pathway were then grouped to calculate module expression score, through AddModuleScore in Seurat function (v3) (Stuart et al., 2019).

\section{Calculating features correlation}

We selected Pearson's correlation to calculate a simple linear correlation between two features. Selected features which are genes, UMI count was used for the calculation. These two selected features were plotted using the FeatureScatterPlot function from Seurat. The statistical analyses and $p$-values were calculated using cor.test function from stats, $\mathrm{R}$ default package. 


\section{Inferring transcription factor activity via pySCENIC}

We performed regulon activity inference using command line implementation of pySCENIC docker (0.1 I.0) (Van de Sande et al., 2020). The smoothed expression matrix was used as an input. We computed co-expresssion networks between identified transcription factors and the rest of the genes using default options of pyscenic -grn command. The list of transcription factors were provided in Supplementary Table 3. The list of co-expressed genes were then used to identify enriched DNA binding motifs of transcription factors, using the pyscenic -ctx command. The enriched DNA binding was then used to confirm the presences of a transcription factor and the target genes, and filtered out the target genes, which does not have the motif surrounding its transcription factor start sites (TSS). We used the motif database from motifs-v9-nr.mgi-m0.00I-00.0 and cis-target database mm9-tss-centered-10 kb-7species.mc9nr., both provided by cistarget [https://resources.aertslab.org/cistarget/databases/homo_sapiens]. The resulting co-expression of TF-target is then grouped into regulons. The activity of the regulons were computed using AUCells, which calculates the enrichment of a selected gene set according to the ranking to gene expression in a single cell. First, genes in each cell were ranked according to their expression profile. Then, this rank was used to plot a recovery curve of all target genes in the regulon, and then to compute the 'Area Under the Curve' (AUC) as inferred to the regulon activity. We employed the default threshold to compute the area under the curve to top 5 percent of the number of genes in the ranking. The resulting matrix was then integrated into the Seurat object. Finally, using all regulon activities calculated from pySCENIC, we performed a dimension reduction method, using Uniform Manifold Approximation and Projection (UMAP) without scaling and centering. This nonlinear dimension reduction method embedded cells in a 2-dimensional space.

\section{Regulon analysis}

We identified the regulon markers at each stage, using the FindAllMarker function in Seurat. The regulon activity overexpressed in each stage were tested Wilcoxon rank sum test versus the regulon activity from all other stages. To limit the number of significant markers, the threshold of log fold-change was set at 0.1 , and adjusted $p$-value (with Bonferroni correction) at 0.0I. The list of regulon markers in each stage are in Supplementary Table 3. We then compiled target genes list from stages regulon marker to perform Gene Ontology Enrichment analysis through topGO package. The list of target genes markers were tested with a list of genes in 
GO terms through Fisher's exact test. The resulting GO terms were ranked according to Fisher's test $p$-value.

\section{Code availability}

All codes, docker, expression matrix used in the analysis of this report are available at http://www.github.com/PankaewSaran/PSPP_project

\section{Author contributions}

SP and PP planned the study. SP and PP led data curation and performed the data analysis. SP and PP wrote the manuscript.

\section{Acknowledgements}

We would like to thank Bo Xia and Dr. Itai Yanai for providing the scRNA expression dataset with metadata for our study.

\section{Competing interests}

The authors declare no competing interests.

\section{References}

Aymard, F., Bugler, B., Schmidt, C. K., Guillou, E., Caron, P., Briois, S., lacovoni, J. S., Daburon, V., Miller, K. M., Jackson, S. P., et al. (20l4). Transcriptionally active chromatin recruits homologous recombination at DNA double-strand breaks. Nat. Struct. Mol. Biol. 2 I, 366-374.

Batenburg, N. L., Thompson, E. L., Hendrickson, E. A. and Zhu, X.-D. (2015). Cockayne syndrome group $B$ protein regulates DNA double-strand break repair and checkpoint activation. EMBO J. 34, I399-14I6.

Bee, L., Fabris, S., Cherubini, R., Mognato, M. and Celotti, L. (20I3). The efficiency of homologous recombination and non-homologous end joining systems in repairing double-strand breaks during cell cycle progression. PLoS One 8, e6906I.

Cha, Y., Heo, S.-H., Ahn, H.-J., Yang, S. K., Song, J.-H., Suh, W. and Park, K.-S. (20I3). Tcea3 regulates the vascular differentiation potential of mouse embryonic stem cells. Gene Expr. 16, 25-30.

Chakraborty, A., Tapryal, N., Venkova, T., Horikoshi, N., Pandita, R. K., Sarker, A. H., Sarkar, P. S., Pandita, T. K. and Hazra, T. K. (2016). Classical non-homologous end-joining pathway utilizes nascent 
RNA for error-free double-strand break repair of transcribed genes. Nat. Commun. 7, 13049.

Derbyshire, M. K., Epstein, L. H., Young, C. S., Munz, P. L. and Fishel, R. (1994). Nonhomologous recombination in human cells. Mol. Cell. Biol. I4, 156-169.

Gomez-Herreros, F. and de Miguel-Jimenez, L. (20I2). TFIIS is required for the balanced expression of the genes encoding ribosomal components under transcriptional stress. Nucleic acids.

Grasser, M., Kane, C. M., Merkle, T., Melzer, M., Emmersen, J. and Grasser, K. D. (2009). Transcript elongation factor TFIIS is involved in arabidopsis seed dormancy. J. Mol. Biol. 386, 598-6II.

Guglielmi, B., Soutourina, J., Esnault, C. and Werner, M. (2007). TFIIS elongation factor and Mediator act in conjunction during transcription initiation in vivo. Proc. Natl. Acad. Sci. U. S. A. 104, 16062-16067.

Guo, J., Grow, E. J., Mlcochova, H., Maher, G. J., Lindskog, C., Nie, X., Guo, Y., Takei, Y., Yun, J., Cai, L., et al. (20|8). The adult human testis transcriptional cell atlas. Cell Res. 28, I|4|-I I57.

Horiuchi, K., Perez-Cerezales, S., Papasaikas, P., Ramos-lbeas, P., López-Cardona, A. P., Laguna-Barraza, R., Fonseca Balvís, N., Pericuesta, E., Fernández-González, R., Planells, B., et al. (2018). Impaired Spermatogenesis, Muscle, and Erythrocyte Function in UI 2 Intron Splicing-Defective Zrsrl Mutant Mice. Cell Rep. 23, I43-I55.

Ito, T., Arimitsu, N., Takeuchi, M., Kawamura, N., Nagata, M., Saso, K., Akimitsu, N., Hamamoto, H., Natori, S., Miyajima, A., et al. (2006). Transcription elongation factor S-Il is required for definitive hematopoiesis. Mol. Cell. Biol. 26, 3194-3203.

Kalogeraki, V. S., Tornaletti, S., Cooper, P. K. and Hanawalt, P. C. (2005). Comparative TFIIS-mediated transcript cleavage by mammalian RNA polymerase II arrested at a lesion in different transcription systems. DNA Repair 4, 1075-1087.

Kettenberger, H., Armache, K.-J. and Cramer, P. (2003). Architecture of the RNA polymerase II-TFIIS complex and implications for mRNA cleavage. Cell I I4, 347-357.

Kim, B., Nesvizhskii, A. I., Rani, P. G., Hahn, S., Aebersold, R. and Ranish, J. A. (2007). The transcription elongation factor TFIIS is a component of RNA polymerase II preinitiation complexes. Proc. Natl. Acad. Sci. U. S. A. 104, 16068-16073.

Kuraoka, I., Suzuki, K., Ito, S., Hayashida, M., Kwei, J. S. M., Ikegami, T., Handa, H., Nakabeppu, Y. and Tanaka, K. (2007). RNA polymerase II bypasses 8-oxoguanine in the presence of transcription elongation factor TFIIS. DNA Repair 6, 84I-85I.

Labhart, P. and Morgan, G. T. (1998). Identification of Novel Genes Encoding Transcription Elongation Factor TFIIS (TCEA) in Vertebrates: Conservation of Three Distinct TFIIS Isoforms in Frog, Mouse, and Human. Genomics 52, 278-288.

Lennon, J. C., 3rd, Wind, M., Saunders, L., Hock, M. B. and Reines, D. (1998). Mutations in RNA polymerase II and elongation factor SIl severely reduce mRNA levels in Saccharomyces cerevisiae. Mol. Cell. Biol. 18, 577I-5779.

Li, X. Z., Roy, C. K., Dong, X., Bolcun-Filas, E., Wang, J., Han, B. W., Xu, J., Moore, M. J., Schimenti, 
J. C., Weng, Z., et al. (20/3). An ancient transcription factor initiates the burst of piRNA production during early meiosis in mouse testes. Mol. Cell 50, 67-8I.

Lisica, A., Engel, C., Jahnel, M., Roldán, É., Galburt, E. A., Cramer, P. and Grill, S. W. (2016). Mechanisms of backtrack recovery by RNA polymerases I and II. Proc. Natl. Acad. Sci. U. S. A. I I3, 2946-2951.

Mao, Z., Bozzella, M., Seluanov, A. and Gorbunova, V. (2008). Comparison of nonhomologous end joining and homologous recombination in human cells. DNA Repair 7, 1765-177I.

Ochiai, H., Hayashi, T., Umeda, M., Yoshimura, M., Harada, A., Shimizu, Y., Nakano, K., Saitoh, N., Liu, Z., Yamamoto, T., et al. (2020). Genome-wide kinetic properties of transcriptional bursting in mouse embryonic stem cells. Sci Adv 6, eaaz6699.

Osorio, D. and Cai, J. J. (2020). Systematic determination of the mitochondrial proportion in human and mice tissues for single-cell RNA-sequencing data quality control. Bioinformatics.

Park, K.-S., Cha, Y., Kim, C.-H., Ahn, H.-J., Kim, D., Ko, S., Kim, K.-H., Chang, M.-Y., Ko, J.-H., Noh, Y.-S., et al. (20I3). Transcription elongation factor Tcea3 regulates the pluripotent differentiation potential of mouse embryonic stem cells via the Leftyl-Nodal-Smad2 pathway. Stem Cells 31, 282-292.

Prather, D. M., Larschan, E. and Winston, F. (2005). Evidence that the elongation factor TFIIS plays a role in transcription initiation at GALI in Saccharomyces cerevisiae. Mol. Cell. Biol. 25, 2650-2659.

Reines, D., Chamberlin, M. J. and Kane, C. M. (1989). Transcription elongation factor SII (TFIIS) enables RNA polymerase II to elongate through a block to transcription in a human gene in vitro. J. Biol. Chem. 264, 10799-10809.

Rhee, S. Y., Wood, V., Dolinski, K. and Draghici, S. (2008). Use and misuse of the gene ontology annotations. Nat. Rev. Genet. 9, 509-5I5.

Schweikhard, V., Meng, C. A., Murakami, K., Kaplan, C. D., Kornberg, R. D. and Block, S. M. (20|4a). TFIIF and TFIIS Enhance the Mechanical Persistence of Transcript Elongation by RNA Polymerase II. Biophysical Journal I06, 486a.

Schweikhard, V., Meng, C., Murakami, K., Kaplan, C. D., Kornberg, R. D. and Block, S. M. (20|4b). Transcription factors TFIIF and TFIIS promote transcript elongation by RNA polymerase II by synergistic and independent mechanisms. Proc. Natl. Acad. Sci. U. S. A. I I I, 6642-6647.

Shema, E., Kim, J., Roeder, R. G. and Oren, M. (20II). RNF20 inhibits TFIIS-facilitated transcriptional elongation to suppress pro-oncogenic gene expression. Mol. Cell 42, 477-488.

Shrivastav, M., De Haro, L. P. and Nickoloff, J. A. (2008). Regulation of DNA double-strand break repair pathway choice. Cell Res. 18, I34-147.

Sigurdsson, S., Dirac-Svejstrup, A. B. and Svejstrup, J. Q. (20I0). Evidence that transcript cleavage is essential for RNA polymerase II transcription and cell viability. Mol. Cel/ 38, 202-2I0.

Sladitschek, H. L., Fiuza, U.-M., Pavlinic, D., Benes, V., Hufnagel, L. and Neveu, P. A. (2020). MorphoSeq: Full Single-Cell Transcriptome Dynamics Up to Gastrulation in a Chordate. Cell I 8 I, 
922-935.e2I.

Stuart, T., Butler, A., Hoffman, P., Hafemeister, C., Papalexi, E., Mauck, W. M., 3rd, Hao, Y., Stoeckius, M., Smibert, P. and Satija, R. (2019). Comprehensive Integration of Single-Cell Data. Cell 177, 1888-1902.e2I.

Svejstrup, J. Q. (2002). Mechanisms of transcription-coupled DNA repair. Nat. Rev. Mol. Cell Biol. 3, 2 I-29.

Van de Sande, B., Flerin, C., Davie, K., De Waegeneer, M., Hulselmans, G., Aibar, S., Seurinck, R., Saelens, W., Cannoodt, R., Rouchon, Q., et al. (2020). A scalable SCENIC workflow for single-cell gene regulatory network analysis. Nat. Protoc. 15, 2247-2276.

Weaver, Z. A. and Kane, C. M. (1997). Genomic characterization of a testis-specific TFIIS (TCEA2) gene. Genomics 46, 516-519.

Xia, B., Yan, Y., Baron, M., Wagner, F., Barkley, D., Chiodin, M., Kim, S. Y., Keefe, D. L., Alukal, J. P., Boeke, J. D., et al. (2020). Widespread Transcriptional Scanning in the Testis Modulates Gene Evolution Rates. Cell I80, 248-262.e2I.

Yang, T., Cui, H., Wen, M., Zuber, J., Kogan, S. C. and Wei, G. (20I8). TCEAI regulates the proliferative potential of mouse myeloid cells. Exp. Cell Res. 370, 55I-560.

Zatreanu, D., Han, Z., Mitter, R., Tumini, E., Williams, H., Gregersen, L., Dirac-Svejstrup, A. B., Roma, S., Stewart, A., Aguilera, A., et al. (2019). Elongation Factor TFIIS Prevents Transcription Stress and R-Loop Accumulation to Maintain Genome Stability. Mol. Cell 76, 57-69.e9. 